\title{
Acute-care hospital reencounters in COVID-19 patients
}

\author{
Jeffrey R. Clark (D) Ayush Batra (D) Nathan A. Shlobin (D) \\ Steven C. Hoffman (i) Z Zachary S. Orban • Igor J. Koralnik (1) • \\ Eric M. Liotta
}

Received: 24 February 2021 / Accepted: 23 April 2021 / Published online: 21 May 2021

(C) American Aging Association 2021

\begin{abstract}
Acute-care hospital reencounters (ACHEs)—encompassing emergency department visits, observation stays, and hospital readmissionsfollowing COVID-19 hospitalization may exacerbate health care system strain and impair recovery from illness. We sought to characterize these reencounters and factors associated with reencounters. We identified the first consecutive 509 patients hospitalized for COVID-19 within an IL hospital network, and examined ACHEs, experienced within 30 days and 4 months of index hospitalization. We identified independent predictors of reencounter using binary logistic regression. Of 509 patients, 466 (91.6\%) were discharged alive from index COVID-19 hospitalization. Within 30 days and 4 months, $12.4 \%$ and $21.5 \%$ of patients, respectively, experienced ACHEs. The median time to first ACHE was 24.2 (IQR 6.5, 55) days. COVID-19 symptom exacerbation was the leading reason for early ACHE (44.8\%). Reencounters, both within 30 days and 4 months, were associated with a history of a neurological disorder before COVID-19 (OR 2.78 [95\% CI 1.53, 5.03]
\end{abstract}

Jeffrey R. Clark and Ayush Batra contributed equally as co-first authors

J. R. Clark - A. Batra - N. A. Shlobin - S. C. Hoffman · Z. S. Orban · I. J. Koralnik · E. M. Liotta $(\bowtie)$

Ken and Ruth Davee Department of Neurology, Northwestern University Feinberg School of Medicine, 625 N. Michigan Ave, Suite 1150, Chicago IL 60630, USA e-mail: Eric.Liotta@ northwestern.edu and OR 2.75 [95\% CI 1.67, 4.53], respectively). Older patients and those with diabetes mellitus, chronic obstructive pulmonary disease, or organ transplantation tended towards more frequent ACHEs. Steroid treatment during COVID-19 hospitalization demonstrated reduced odds of 30-day reencounter (OR 0.31 [95\% CI 0.091, 0.79]). Forty-nine patients had repeat SARS-CoV-2 nasopharyngeal testing during a reencounter; twelve $(24.5 \%)$ patients had positive reencounter tests and experienced more frequent reencounters than those testing negative. COVID-19 symptom exacerbation is a leading cause of early ACHE after COVID-19 hospitalization, and steroid use during index hospitalization may reduce early reencounters. Neurologic illness before COVID-19 predicts ACHEs.

Keywords COVID-19 - Reencounter .

Readmission $\cdot$ Post-viral syndrome $\cdot$ Post-hospital syndrome

\section{Introduction}

As of February 2021, the severe acute respiratory syndrome coronavirus 2 (SARS-CoV-2) has caused over 27.8 million cases of coronavirus disease 2019 (COVID-19) and 500,000 deaths in the USA [1]. Moreover, there are greater than 228 hospitalizations for COVID-19 per 100,000 people in the USA, which has 
strained the capacity and resources of the health care system [2-4].

Acute-care hospital reencounters (ACHEs) - encompassing hospital readmissions, visits to the emergency department (ED), and observation unit stays - can compound the strain on health care system resources due to an initial hospitalization and may impair recovery from illness and optimization of patient outcome, especially among elderly patients [5-9]. ACHEs may also provide insight into the ongoing health challenges of patients following COVID-19 by identifying symptoms impairing health to the point of requiring acute medical attention. Multiple studies have suggested that the sequelae of COVID-19 may persist beyond the initial bout of respiratory illness $[10,11]$. Persistent symptoms including fatigue, dyspnea, headache, and cognitive slowing have been reported by "long hauler" patients for weeks or even months after their acute COVID-19 illness, irrespective of whether they were initially hospitalized for COVID19 [10-13]. While there have been reports of persistent post-COVID-19 symptoms leading to presentation to EDs or hospital admission [12], there are limited data regarding the frequency of and reasons for ACHEs in the months following hospitalization for COVID-19.

This study characterizes the rate of short- and longterm ACHEs in a consecutive cohort of patients both within 30 days and within 4 months following COVID19 hospitalization. Thirty days was selected for shortterm ACHE as that time period represents a standard time metric for tracking short-term reencounters after hospitalization. Four months after COVID-19 hospitalization was selected for long-term ACHE as it represented the longest follow-up available for all patients in our cohort at the time of data collection. We also sought to elucidate the reasons and risk factors for ACHEs. This may assist in efforts to minimize avoidable reencounters as well as provide insight into the ongoing health care challenges experienced by COVID-19 patients following their initial hospitalization.

\section{Methods}

Study design, setting, and subjects

To identify patients with an initial hospitalization for COVID-19, we used a computerized query of the electronic medical record to determine the first consecutive patients admitted with a confirmed primary diagnosis of COVID-19 to the Northwestern Medicine Healthcare (NMHC) system between 5 March and 6 April 2020, which resulted in a convenience sample of 509 consecutive patients. NMHC consists of one academic medical center and nine other hospitals in the Chicago, IL, area. COVID-19 diagnosis was confirmed by SARS-CoV-2 reverse transcription polymerase chain reaction (RT-PCR) assay of nasopharyngeal swab or bronchoalveolar lavage fluid. All laboratory and radiologic assessments were performed as part of routine clinical care. The study was approved by our institutional review board (STU00212627) with waiver of consent for retrospective analysis.

Measurements and outcomes

ACHEs included ED visits, observation stays, or hospital readmissions. We excluded all scheduled reencounters and reencounters pertaining to labor and delivery or accidental trauma. For the analysis of ACHEs, we excluded patients who died during the initial COVID-19 hospitalization. ACHEs following the index hospitalization were identified by query of the NMHC system electronic medical record and by query for reencounters at any of the 20 participating IL hospital systems in the Epic (Epic Systems Corporation, Verona, WI, USA) Care Everywhere Network. We included ED visits and observation stays in addition to hospital readmissions given the literature, suggesting that efforts to reduce hospital readmission in the USA may have shifted a greater portion of acute care to treat-anddischarge visits in the ED and observations stays [5].

Demographic, medical comorbidity, prehospitalization medication usage, and hospital course data were collected by electronic medical record review, as previously described [14]. Medications received during initial hospitalization, including steroids, were collected by automated electronic query. Given prominent reports of headaches and "brain fog" as persistent post-COVID-19 symptoms and our prior report of frequent neurologic manifestations among hospitalized COVID-19 patients [12, 14], we included data on the incidence of neurologic manifestations during patients' COVID-19 disease course in our analysis of potential factors contrib- 
uting to reencounters. Neurologic manifestations were identified by neurologist adjudication, which excluded neurologic manifestations that resulted from medication effects, as previously described [14]. The primary reason for each reencounter was identified by review of physician-documented clinical notes and reported as determined by the attending physician of record for the reencounter. We also identified whether patients with a reencounter had a repeat SARS-CoV-2 RT-PCR assay performed at any point during the reencounter. Patients were categorized as having a positive reencounter RT-PCR if they had a positive result during any reencounter. In the case of multiple reencounter RT-PCR assays, the date of reencounter assay was recorded as the last available positive reencounter RT-PCR or the first available negative reencounter RT-PCR if a patient never had a positive reencounter RT-PCR result. Our RT-PCR assay identifies the presence of SARS-CoV-2 RNA but does not identify whether that RNA is replicative.

\section{Statistical analysis}

Data were summarized as the number of patients/ events (frequency), mean (standard deviation) for normally distributed variables, and median (interquartile range (IQR)) for non-normally distributed variables. Associations were assessed using Fisher's exact test, Spearman's rank correlation test, and Wilcoxon rank-sum test. Binary logistic regression models were developed to identify adjusted factors associated with (1) ACHE within 30 days of discharge from index hospitalization and (2) ACHE within 4 months of discharge from index hospitalization. In each case, we first developed a model using a priori variables of age and sex, which are factors widely appreciated as related to COVID-19 disease severity. Given relatively limited data in the literature regarding additional factors associated with ACHE after COVID-19 hospitalization, we then included additional variables in the model that were univariately associated with ACHE at $p \leq 0.15$ and not highly collinear with a variable already included in the model. To avoid overfitting models to the data, we used a backward variable selection algorithm based on Akaike information criterion optimization to develop final parsimoniously adjusted models of ACHE within 30 days and within 4 months. Two-sided $p \leq 0.05$ was considered sig- nificant, and all analyses were performed in $\mathrm{R}$ version 3.5.0 (R Foundation for Statistical Computing, Vienna, Austria).

\section{Results}

Study subject characteristics

Of 509 patients hospitalized with COVID-19 between March 5, 2020 and April 6, 2020, 466 (91.6\%; 250 [53.6\%] males, 57.4 [16.5] years old) were discharged alive. ACHEs occurred in 58 (12.4\%) patients within 30 days of index hospitalization discharge, and an additional $42(9.0 \%)$ patients experienced their first ACHE between 31 days and 4 months of index hospitalization discharge (100 [21.5\%] total patients with ACHEs within 4 months). Among those with an ACHE, the median number of ACHEs experienced within 4 months was $1(1,2)$ encounter per patient. Table 1 summarizes the demographic features of the cohort stratified by hospital reencounter status. Neither age, sex, race, nor ethnicity was associated with ACHEs, either at 30 days or 4 months. The only comorbidity associated with ACHEs was a history of neurological disorders prior to COVID-19 (43.1\% vs. $22.8 \%$ for reencounters within 30 days, $p=0.002$; $41.0 \%$ vs. $21.0 \%$ for reencounters within 4 months, $p<0.001$ ), though patients with diabetes mellitus or chronic obstructive pulmonary disease had a tendency towards more frequent reencounters within 4 months. The past medical history of neurological disorders in this cohort included spinal stenosis, radiculopathy, epilepsy, prior stroke, neurodegenerative diseases including dementia, history of migraines, and peripheral neuropathy. Patients with ACHEs had a tendency towards shorter time from COVID-19 symptom onset to index hospitalization (non-significant tendency for reencounters within 30 days: 6.0 [2.1, 9.75] vs. 7.0 $[4.0,10.0], p=0.096$; significant difference for reencounters within 4 months: 5.5 [2.3, 8.25] vs. 7.0 [4.0, $10.0], p=0.006$ ). Neither a need for mechanical ventilation during the COVID-19 hospitalization, length of index hospitalization, nor functional outcome at index hospitalization discharge was associated with ACHEs, within either 30 days or 4 months. Patients with reencounters within 30 days had less frequently received steroids during their COVID-19 hospitalization $(6.9 \%$ vs. $18.6 \%, p=0.042)$; however, there was 


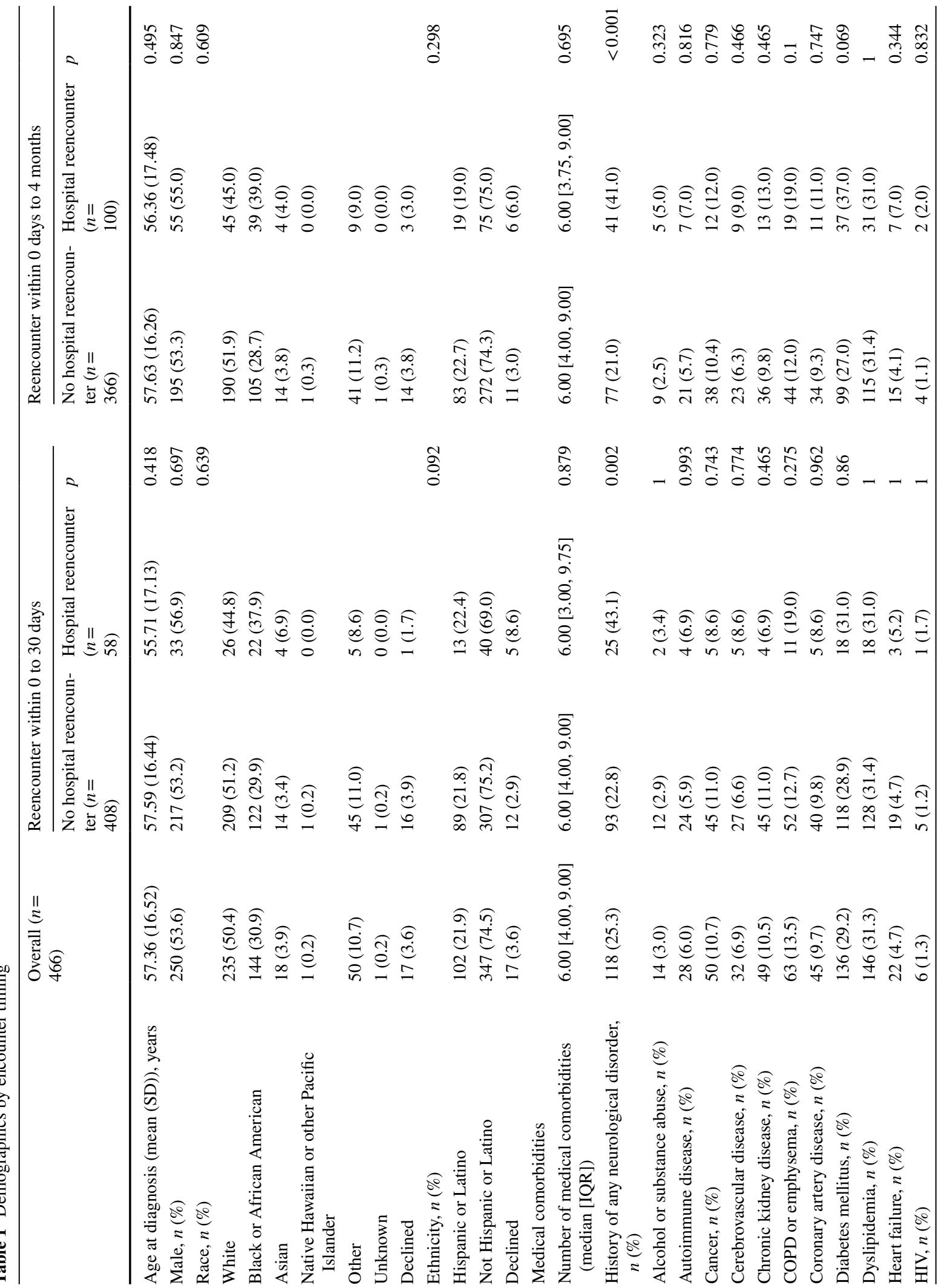




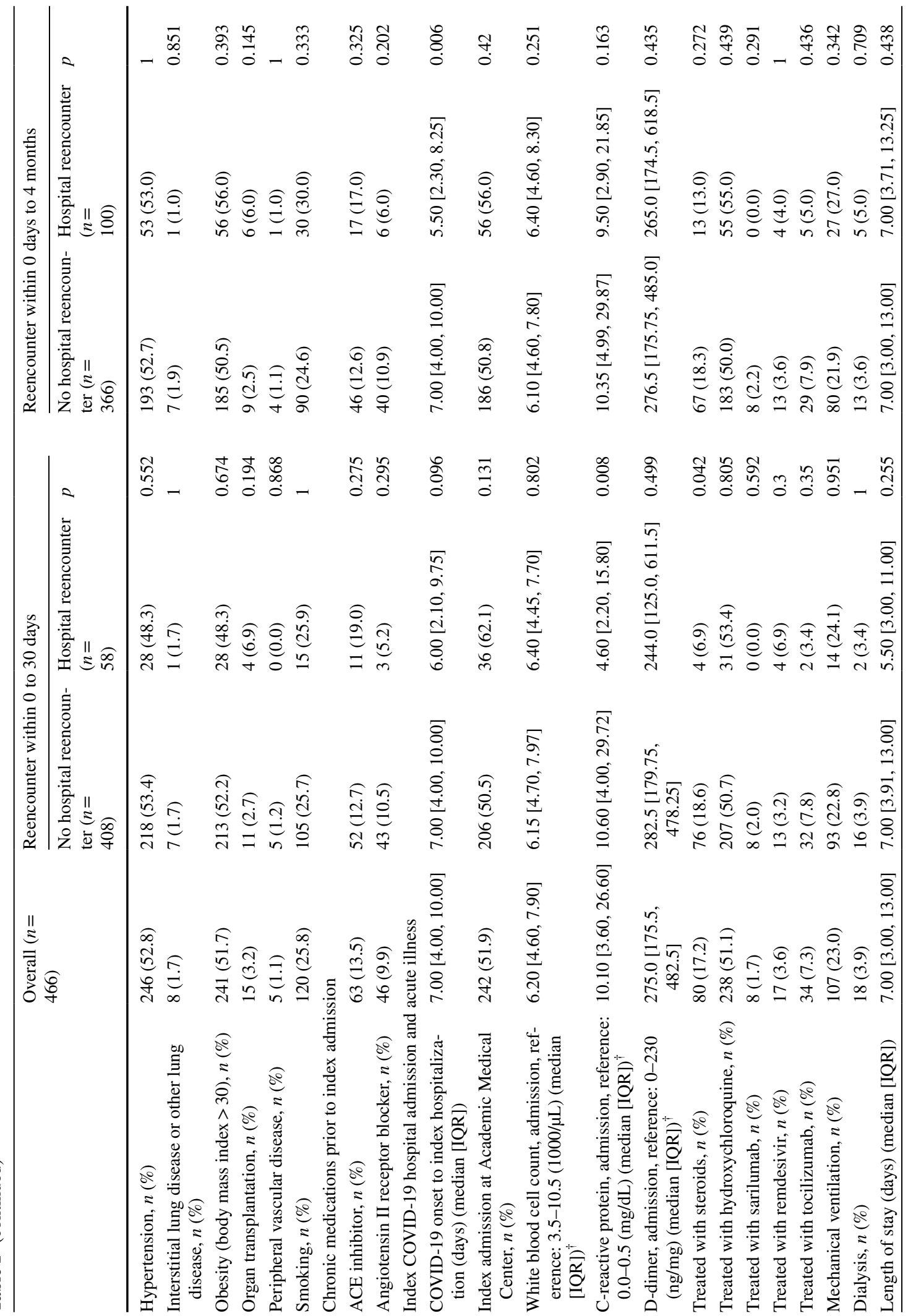




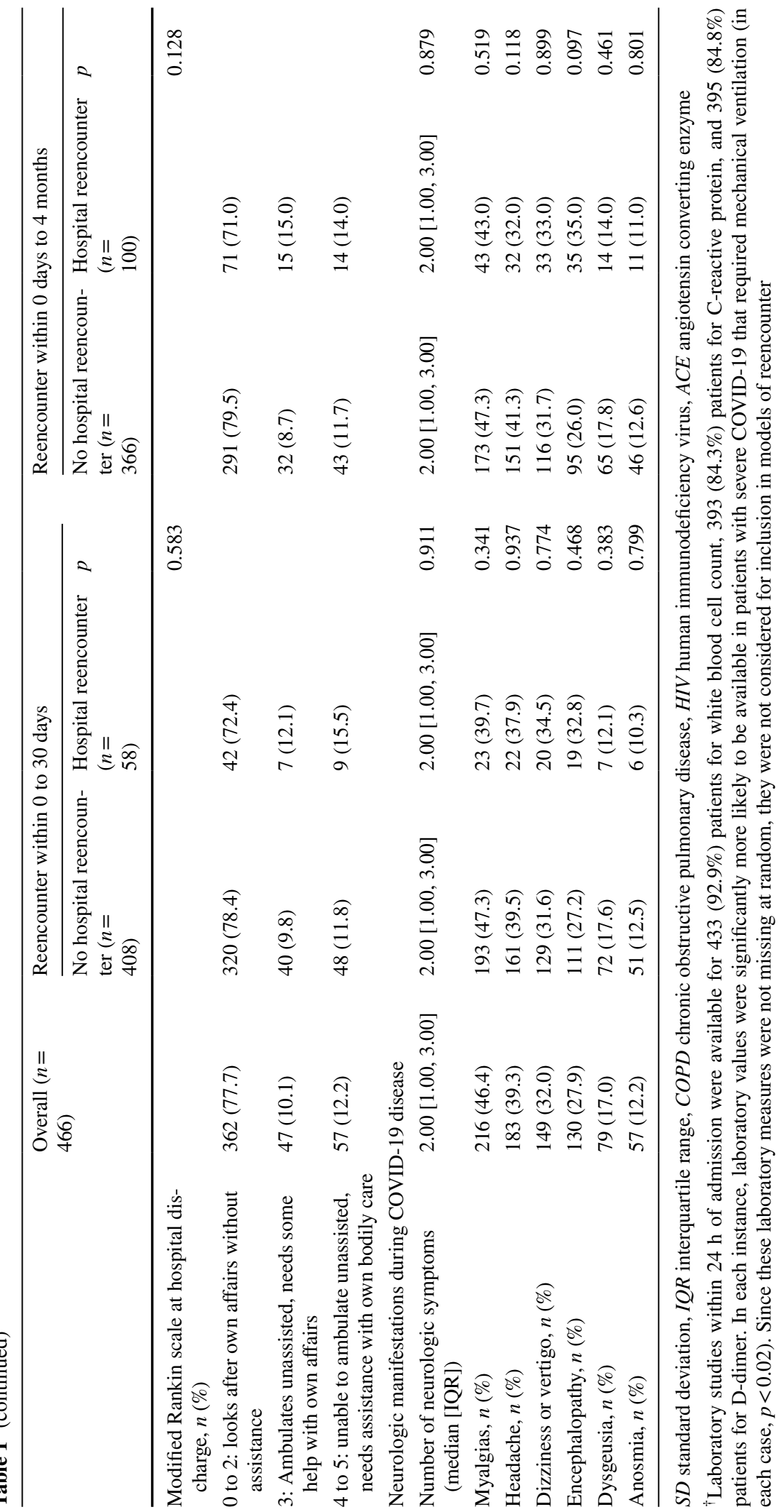


no association between steroids and reencounters within 4 months. In addition, there was a non-significant tendency towards more frequent encephalopathy during the COVID-19 hospitalization in those who had reencounters within 4 months (35\% vs. $26 \%$, $p=0.097)$.

Of the 100 patients who experienced an ACHE, 49 had at least one SARS-CoV-2 RT-PCR assay performed during a reencounter. Of these, 12 (24.5\%) patients had a positive SARS-CoV-2 RT-PCR during a reencounter, including four patients who had a previously documented negative repeat RT-PCR. The time from index hospital admission to the reencounter RT-PCR did not differ significantly between those with positive versus negative assay results (62.8 [28.0, 103.25] vs. 73.9 [56.25, 96.25] days, $p=0.42)$. Five $(42 \%)$ of the 12 patients with positive reencounter RT-PCR assays were positive more than 3 months after hospitalization for COVID-19; the positive assay furthest from index hospitalization occurred at 124 days, during a hospital readmission that started within 4 months. In addition, patients with a positive reencounter RT-PCR experienced more ACHEs within 4 months than those who only had negative reencounter RT-PCR assays (2 [2.0,3.25] vs. 1 [1.0,2.0], $p=0.002)$, although there was no difference in the length of index COVID-19 hospitalization, frequency of mechanical ventilation during index hospitalization, or the time between discharge from the index hospitalization and the first ACHE. Only 2 patients with a positive reencounter SARS-CoV-2 RT-PCR experienced a single ACHE. The reasons for the first ACHE in these 12 positive reencounter RT-PCR patients included COVID19 exacerbation (2), bleeding (2), encephalopathy (1), ischemic stroke (1), deep venous thrombosis (1), bacterial pneumonia (1), infection other than COVID-19 or pneumonia (1), acute renal dysfunction (1), heart failure (1), and stridor and dyspnea due to tracheal stenosis (1).

Features of acute-care hospital reencounters

A total of 67 ACHEs among 58 patients occurred within 30 days of discharge, and an additional 85 ACHEs among 65 patients occurred within 31 days to 4 months of discharge. The median time to first ACHE from index COVID-19 hospitalization discharge was $24.2(6.5,55)$ days. Among reencounters within 30 days, $28(41.8 \%)$ were hospital readmissions, $12(17.9 \%)$ were observations stays, and 27 (40.3\%) were ED visits. Among reencounters between 31 days and 4 months, 41 (48.2\%) were hospital readmissions, 7 (8.2\%) were observations stays, and 37 (43.5\%) were ED visits. Two deaths occurred in readmitted patients, both of whom returned within 30 days after index hospitalization with COVID-19 pulmonary symptom exacerbation requiring respiratory support.

Table 2 summarizes the reasons for ACHE stratified by timing and type of reencounter, noting that there were patients who experienced multiple reencounters during both 30-day and 31-day to 4-month time periods. Respiratory complaints contributed to $38(56.7 \%)$ of 30-day ACHEs. The most frequent reason for 30-day ACHE was exacerbation of COVID19 symptoms, including dyspnea and fatigue, which accounted for $30(44.8 \%)$ ACHEs in 29 patients. Of these, COVID-19 symptom exacerbation without respiratory distress occurred in 24 (35.8\%) ACHEs while respiratory distress requiring supportive care occurred in 6 (9\%) ACHEs. Among the 29 patients with an ACHE for COVID-19 symptom exacerbation, none had received steroids during their index hospitalization; in contrast, among the 437 remaining patients who did not have a 30-day ACHE for COVID-19 symptom exacerbation, 80 (18.3\%) had received steroids during their index hospitalization $(p=0.005)$. The next most frequent reasons for reencounters within 30 days were infections besides COVID-19 or pneumonia (5 [7.5\%]), bleeding (5 [7.5\%]), bacterial pneumonia (4 [6.0\%]), and other pulmonary issues (4 [6.0\%]).

Respiratory complaints contributed to 15 (17.6\%) ACHEs between 31 days and 4 months. The most frequent reasons for ACHEs between 31 days and 4 months were pulmonary issues excluding bacterial pneumonia (11 [12.9\%]; including asthma or chronic obstructive pulmonary disease exacerbation, pulmonary edema/effusion, pleurisy, and airway stenosis), pain besides abdominal or chest pain (9 [10.6\%]), acute renal dysfunction (7 [8.2\%]), and abdominal pain (6 [7.1\%]). After 30 days, ACHE for COVID-19 symptom exacerbation occurred in only 4 emergency room visits out of 85 (4.7\%) ACHEs. 
Table 2 Reason for hospital reencounters

\begin{tabular}{|c|c|c|c|c|c|c|}
\hline & \multicolumn{3}{|c|}{ Within 0 to 30 days $^{\dagger}$} & \multicolumn{3}{|c|}{ Within 31 days to 4 months $^{\dagger}$} \\
\hline & $\begin{array}{l}\text { Hospital read- } \\
\text { mission }(n= \\
28)\end{array}$ & $\begin{array}{l}\text { Observation } \\
\text { stay }(n= \\
12)\end{array}$ & $\begin{array}{l}\text { Emergency } \\
\text { room }(n= \\
27)\end{array}$ & $\begin{array}{l}\text { Hospital } \\
\text { readmission } \\
(n= \\
41)\end{array}$ & $\begin{array}{l}\text { Observation s } \\
\text { tay }(n= \\
7)\end{array}$ & $\begin{array}{l}\text { Emergency } \\
\text { room }(n= \\
37)\end{array}$ \\
\hline \multicolumn{7}{|l|}{ Primary encounter reason, $n(\%)$} \\
\hline $\begin{array}{l}\text { COVID-19 exacerbation, without } \\
\text { respiratory distress }\end{array}$ & $2(7.1)$ & $6(50.0)$ & $16(59.3)$ & $0(0.0)$ & $0(0.0)$ & $4(10.8)$ \\
\hline $\begin{array}{l}\text { COVID-19 exacerbation, with respira- } \\
\text { tory distress }\end{array}$ & $6(21.4)$ & $0(0.0)$ & $0(0.0)$ & $0(0.0)$ & $0(0.0)$ & $0(0.0)$ \\
\hline $\begin{array}{l}\text { Pulmonary issue (including asthma } \\
\text { or COPD exacerbation, pulmonary } \\
\text { edema/effusion, pleurisy, and airway } \\
\text { stenosis) }\end{array}$ & $4(14.3)$ & $0(0.0)$ & $0(0.0)$ & $8(19.5)$ & $0(0.0)$ & $3(8.1)$ \\
\hline Bacterial pneumonia & $4(14.3)$ & $0(0.0)$ & $0(0.0)$ & $3(7.3)$ & $1(14.3)$ & $0(0.0)$ \\
\hline \multicolumn{7}{|l|}{ Neurological } \\
\hline Encephalitis & $1(3.6)$ & $0(0.0)$ & $0(0.0)$ & $0(0.0)$ & $0(0.0)$ & $0(0.0)$ \\
\hline Encephalopathy/delirium & $0(0.0)$ & $0(0.0)$ & $1(3.7)$ & $1(2.4)$ & $0(0.0)$ & $0(0.0)$ \\
\hline Seizure & $0(0.0)$ & $1(8.3)$ & $0(0.0)$ & $0(0.0)$ & $0(0.0)$ & $1(2.7)$ \\
\hline Stroke or transient ischemic attack & $0(0.0)$ & $0(0.0)$ & $0(0.0)$ & $5(12.2)$ & $0(0.0)$ & $0(0.0)$ \\
\hline Neurologic, other & $0(0.0)$ & $1(8.3)$ & $1(3.7)$ & $0(0.0)$ & $1(14.3)$ & $4(10.8)$ \\
\hline \multicolumn{7}{|l|}{ Cardiac } \\
\hline Heart failure & $1(3.6)$ & $0(0.0)$ & $0(0.0)$ & $4(9.8)$ & $0(0.0)$ & $0(0.0)$ \\
\hline Cardiac, other & $0(0.0)$ & $0(0.0)$ & $0(0.0)$ & $2(4.9)$ & $1(14.3)$ & $2(5.4)$ \\
\hline \multicolumn{7}{|l|}{ Other } \\
\hline Acute renal dysfunction & $1(3.6)$ & $0(0.0)$ & $1(3.7)$ & $6(14.6)$ & $0(0.0)$ & $1(2.7)$ \\
\hline Abdominal pain & $0(0.0)$ & $1(8.3)$ & $0(0.0)$ & $0(0.0)$ & $1(14.3)$ & $5(13.5)$ \\
\hline Anxiety & $0(0.0)$ & $0(0.0)$ & $2(7.4)$ & $0(0.0)$ & $0(0.0)$ & $1(2.7)$ \\
\hline Bleeding & $4(14.3)$ & $0(0.0)$ & $1(3.7)$ & $3(7.3)$ & $0(0.0)$ & $2(5.4)$ \\
\hline Fatigue & $1(3.6)$ & $0(0.0)$ & $1(3.7)$ & $0(0.0)$ & $0(0.0)$ & $1(2.7)$ \\
\hline $\begin{array}{l}\text { Infection other than COVID-19 or } \\
\text { pneumonia }\end{array}$ & $2(7.1)$ & $1(8.3)$ & $2(7.4)$ & $5(12.2)$ & $0(0.0)$ & $1(2.7)$ \\
\hline Non-cardiac chest pain & $0(0.0)$ & $0(0.0)$ & $1(3.7)$ & $0(0.0)$ & $0(0.0)$ & $4(10.8)$ \\
\hline Pain, other & $0(0.0)$ & $0(0.0)$ & $1(3.7)$ & $0(0.0)$ & $1(14.3)$ & $4(10.8)$ \\
\hline PE or DVT & $0(0.0)$ & $1(8.3)$ & $0(0.0)$ & $2(4.9)$ & $0(0.0)$ & $1(2.7)$ \\
\hline Other & $2(7.1)$ & $1(8.3)$ & $0(0.0)$ & $2(4.9)$ & $2(28.6)$ & $3(8.1)$ \\
\hline
\end{tabular}

$C O P D$ chronic obstructive pulmonary disease, $P E$ pulmonary embolism, $D V T$ deep vein thrombosis

${ }^{\dagger}$ Note that some patients experienced multiple reencounters during each time period

Factors independently associated with reencounter

In adjusted models (Table 3), a history of a neurologic disorder prior to COVID-19 was independently associated with increased odds of a 30-day ACHE (OR 2.78; 95\% CI 1.53, 5.03; $p<0.001$ ) while receiving steroids during the index hospitalization was associated with reduced odds of a 30-day ACHE (OR 0.31; 95\% CI 0.09, 0.79; $p=0.029$ ). In adjusted models of ACHE within 4 months (Table 4), a history of a neurologic disorder prior to COVID-19 was independently associated with increased odds of an ACHE (OR 2.75; 95\% CI 1.67, 4.53; $p<0.001$ ) while patients who had a longer time from COVID- 
19 symptom onset to index hospitalization demonstrated decreased odds of an ACHE (OR 0.957 per day; 95\% CI 0.916, 0.999; $p=0.048$ ). Older patients and those with diabetes mellitus, chronic obstructive pulmonary disease, or organ transplantation had a non-significant tendency towards greater odds of a reencounters within 4 months. Of note, dichotomizing age at 65 years old, rather than treating age as a continuous variable, had no meaningful impact on the effect estimates of either the 30-day or 4-month adjusted models.

\section{Discussion}

This study demonstrates that within 30 days and within 4 months following COVID-19 hospitalization, $12.4 \%$ and $21.5 \%$ of patients, respectively, experienced ACHEs consisting of ED visits, observation stays, and readmission. Reencounter, within both 30 days and 4 months, was associated with a past medical history of a neurological disorder before COVID-19. Meanwhile, receiving steroid treatment during initial COVID-19 hospitalization reduced the odds of 30-day reencounter, and a longer duration between COVID-19 symptom onset and initial hospitalization was associated with lower likelihood of 4-month reencounter.

Hospital readmissions, in general, have been associated with a number of factors such as age, length of stay, and follow-up care [15-18]. The existing literature on COVID-19 reencounters describes 14-day reencounter rates of $3.6 \%$, most commonly for respiratory complaints and associated with variables including history of hypertension or COPD and shorter initial length of stay [19]; additionally, 30 -day hospital readmission rates of $6.8 \%$ were reported in association with medical comorbidities including hypertension, diabetes, chronic pulmonary disease, liver disease, and cancer [20]. Atalla et al. [20] also showed that readmitted patients were less likely to have required ICU care and intubation during initial COVID-19 hospitalization. In contrast, we found no association between need for mechanical ventilation or initial hospital length of stay, as measures of disease severity, and ACHE. In agreement with these other studies, we found that respiratory complaints, including exacerbation of COVID-19 symptoms, were the leading reason for early ACHEs. The differences in findings between our study and the others in the literature might be related to different durations of reencounter followup, inclusion of ED visits and observations stays in addition to hospital readmissions, and identification of ACHEs at multiple hospital systems in our study (using the EPIC Care Everywhere Network). In addition, regional differences, such as COVID-19 case count and reports of hospital resource availability, may have influenced patients' likelihood to represent to the hospital. Similarly, local health care resources and hospital bed availability may have influenced whether health care providers attempted to address ACHEs by ED visits or observation stays rather than hospitalization. In fact, literature prior to the COVID-19 pandemic suggests there may already have been a growing tendency in the USA to reduce hospital readmissions by shifting a greater portion of acute care to treat-and-discharge visits in the ED and observation stays [5]. Our study was conducted in the Chicago, IL, metropolitan area whereas the two COVID-19 reencounter studies cited above were conducted on the East Coast of the United States, all including COVID-19 hospitalization during March and April of 2020. Unlike systems in other regions of the USA, our health care system was not overwhelmed in March and April of 2020, an observation in part reflected in our markedly lower hospital mortality compared to contemporaneous mortality rates at major health care systems based in New York City (8.4\% vs. $21.4 \%$ hospital mortality) [21]. In fact, it is possible that our study findings are most generalizable to hospital system functioning with residual capacity. It is also noteworthy, and perhaps surprising, that we did not find a significant difference in ACHEs between racial and ethnic groups. While granular socioeconomic data was not available for our cohort, future studies might consider including socioeconomic factors, in addition to race and ethnicity, when investigating disparities in COVID-19 outcomes.

Furthermore, due to growing awareness of and concern for prolonged symptoms and sequelae of COVID-19 [10-12], we expanded our investigation to 4 months in order to offer comparison of shorterversus longer-term patterns in ACHEs. In particular, we noted that COVID-19 respiratory complaints were a common reason for reencounters before 30 days, but this was an infrequent reason after 30 days and 
Table 3 Model of 30-day hospital reencounter

\begin{tabular}{|c|c|c|c|c|c|c|}
\hline \multirow[t]{2}{*}{ Variable } & \multicolumn{3}{|l|}{ Full model } & \multicolumn{3}{|c|}{ Parsimonious model } \\
\hline & Odds ratio & $95 \% \mathrm{CI}$ & $p$ value & Odds ratio & $95 \% \mathrm{CI}$ & $p$ value \\
\hline Age, years & 0.987 & $0.970,1.004$ & 0.148 & 0.987 & $0.970,1.004$ & 0.149 \\
\hline Sex, male & 1.18 & $0.669,2.10$ & 0.570 & - & - & - \\
\hline Past medical history of a neurologic disorder & 2.70 & $1.48,4.91$ & 0.001 & 2.78 & $1.53,5.03$ & $<0.001$ \\
\hline $\begin{array}{l}\text { Admission to Academic Medical Center for } \\
\text { COVID-19 hospitalization }\end{array}$ & 1.55 & $0.872,2.80$ & 0.140 & 1.52 & $0.856,2.73$ & 0.158 \\
\hline $\begin{array}{l}\text { Any steroid treatment during COVID-19 hospi- } \\
\text { talization }\end{array}$ & 0.312 & $0.092,0.800$ & 0.030 & 0.309 & $0.091,0.792$ & 0.029 \\
\hline $\begin{array}{l}\text { Time from COVID-19 symptoms onset to hospi- } \\
\text { talization for COVID, days }\end{array}$ & 0.973 & $0.924,1.02$ & 0.30 & - & - & - \\
\hline
\end{tabular}

A priori variables included age and gender. Full models included a priori variables and additional variables demonstrating the difference between acute hospital encounter and no encounter groups at $p \leq 0.15$. Additional variables were included, provided the variable was not collinear with a factor already in the model with a lower $p$ value. The McKelvey-Zavoina pseudo $r^{2}$ value for the full model was 0.13

did not require hospitalization or observation stay in our cohort after 30 days. Our data suggest that the 30-day window is a higher-risk period for reencounter related to COVID-19 symptom recurrence or exacerbation, as well as the time frame during which these concerns are most likely to reach a severity meriting observation or readmission. In addition, the finding that 30-day ACHEs were largely driven by COVID-19 symptom exacerbation, while 4-month ACHEs were not, offers a plausible explanation for the association we identified between steroid treatment during initial COVID-19 hospitalization and a lower likelihood of subsequent 30-day ACHE but a lack of association with 4-month ACHE. Our cohort size was not large enough to consider whether steroid effects might differ across strata of disease severity, but this could be a consideration for future studies as large multicenter cohorts become more readily available.

In addition, we found that nearly a quarter of patients who had a repeat SARS-CoV-2 RT-PCR assay during a reencounter were positive and that positive RT-PCR assays could occur even longer than 3 months after hospitalization for COVID-19. This frequency of prolonged RT-PCR positivity is consistent with a recently reported rate of $18.2 \%$ but demonstrates a potential for SARS-CoV-2 assays to remain positive longer than the follow-up dura-

Table 4 Model of 4-month acute hospital reencounter

\begin{tabular}{|c|c|c|c|c|c|c|}
\hline \multirow[t]{2}{*}{ Variable } & \multicolumn{3}{|l|}{ Full model } & \multicolumn{3}{|c|}{ Parsimonious model } \\
\hline & Odds ratio & $95 \% \mathrm{CI}$ & $p$ value & Odds ratio & $95 \% \mathrm{CI}$ & $p$ value \\
\hline Age & 0.985 & $0.970,0.999$ & 0.043 & 0.987 & $0.973,1.001$ & 0.070 \\
\hline Sex, male & 1.13 & $0.705,1.82$ & 0.614 & - & - & - \\
\hline Past medical history of a neurologic disorder & 2.69 & $1.62,4.44$ & $<0.001$ & 2.75 & $1.67,4.53$ & $<0.001$ \\
\hline History of diabetes mellitus & 1.55 & $0.938,2.52$ & 0.084 & 1.56 & $0.949,2.55$ & 0.077 \\
\hline History of COPD & 1.83 & $0.960,3.39$ & 0.060 & 1.75 & $0.932,3.20$ & 0.074 \\
\hline History of organ transplantation & 2.60 & $0.809,7.79$ & 0.093 & 2.83 & $0.893,8.41$ & 0.064 \\
\hline $\begin{array}{l}\text { Time from COVID-19 symptoms onset to hospitali- } \\
\text { zation for COVID-19, days }\end{array}$ & 0.961 & $0.919,1.002$ & 0.073 & 0.957 & $0.916,0.999$ & 0.048 \\
\hline Encephalopathy during COVID-19 hospitalization & 1.37 & $0.802,2.30$ & 0.244 & - & - & - \\
\hline
\end{tabular}

A priori variables included age and gender. Full models included a priori variables and additional variables demonstrating the difference between acute hospital encounter and no encounter groups at $p \leq 0.15$. Additional variables were included, provided the variable was not collinear with a factor already in the model with a lower $p$ value. The McKelvey-Zavoina pseudo $r^{2}$ value for the full model was 0.11 
tion available in that report [22]. We also found that patients with a positive reencounter RT-PCR had more reencounters within 4 months than those whose reencounter RT-PCR assays were negative. While this may be related to a greater availability for repeated testing in those with more frequent reencounters, this observation would be consistent with an increased vulnerability to symptoms requiring medical attention in those with ongoing expression of viral material. Others have proposed that such a COVID-19 post-viral syndrome might exist and represents both a long-term public health challenge and a potential contributor to poor quality of life recovery for individual COVID-19 patients [23]. While our data are consistent with the existence of postviral syndrome, such a hypothesis requires specific testing in a larger cohort. If a COVID-19 post-viral syndrome exists, the development of specialized outpatient centers for the management of post-acute COVID-19 might be a means to improve patient recovery while decompressing ACHEs by shifting care to the outpatient setting.

It may seem surprising that a prior history of neurologic disorders before COVID-19 was associated with increased odds of ACHE since respiratory complaints appeared to be a driving force for early reencounters. However, the effects of COVID19 on the nervous system are being increasingly reported [14, 24-29]. We previously demonstrated that encephalopathy, as a neurologic complication of COVID-19 hospitalization, was associated with worse patient functional outcome, and a history of premorbid neurologic disorder was associated with both increased odds of encephalopathy during COVID-19 hospitalization and worse functional outcome [14]. Therefore, premorbid neurologic disease may represent a unique vulnerability in the recovery from COVID-19, which should be investigated further. While we only observed a tendency towards increased 4-month reencounter in those who experienced encephalopathy during their COVID-19 hospitalization, it is possible this was due to inadequate cohort size to detect a statistically significant relationship. Furthermore, the size of our cohort was insufficient to examine an association between ACHE and each subtype of premorbid neurologic disease; as the size of COVID-19 cohorts increases, future studies might examine the associations between specific premorbid neurologic diagnoses and various outcome endpoints following acute COVID-19.

Our study has limitations that should be acknowledged. First, it is possible that some patients experienced ACHEs that were not identified by our study. However, we mitigated this limitation by searching for ACHEs at the 20 IL hospital systems participating in the EPIC Care Everywhere Network. Furthermore, missed ACHEs outside the EPIC network would represent a non-systematic source of bias that would not be expected to alter the distribution of reasons for ACHEs or factors associated with ACHE occurrence, but could lead to an overall underestimation of ACHE frequency by our study. Secondly, ACHEs may underrepresent patients who experienced severe COVID-19 illness due to a propensity for these patients to enter inpatient rehabilitation or nursing facilities following discharge where some medical concerns may have been addressed without return to the hospital. It is also possible that severely ill patients might die from a complication prior to reaching a hospital and would not therefore register as an ACHE. As such, while reencounters illustrate common ongoing, reemergent, or new concerns of COVID-19 patients, they may not be the most informative metric to gauge the medical needs of severely ill COVID-19 patients following discharge. Future research might examine the medical needs experienced by severely affected COVID-19 patients who require inpatient rehabilitation or nursing facility stays. Additionally, fear of reexposure to the virus or increased access to telehealth resources may have prevented some reencounters for issues that typically would have resulted in a reencounter prior to the COVID-19 pandemic. In fact, the novel features of COVID-19 and the considerable impact of the pandemic on health care operations make meaningful comparison to historical reencounter data challenging. Due to the retrospective nature of our study, it is possible that not all risk factors for reencounter were examined. For example, followup care after discharge has been identified as influential for rates of readmission [17, 18], a variable which was not investigated in this study. However, given the novelty of COVID-19 and the unclear natural history after acute illness, it may be more challenging to implement effective post-hospital follow-up recommendations than for more well- 
known diseases. It is also possible that our study was underpowered to detect associations between uncommon comorbidities, such as organ transplantation, and ACHE.

In summary, COVID-19 symptom exacerbations along with other respiratory complaints are leading causes of early ACHE after index COVID-19 hospitalization, and steroid use during index hospitalization may reduce the odds of early ACHEs. A history of neurologic illness before COVID-19 is associated with increased likelihood of ACHE, in addition to worse functional outcome from COVID-19. A potential unique role for neurologic factors in acute illness and recovery from COVID-19 requires further investigation.

Acknowledgements Our author group participates in the Global Consortium Study of Neurologic dysfunction in COVID-19 (GCS-NeuroCOVID).

Author contribution Jeffrey R. Clark collected the patient data; assisted in the data management, data analysis, and study interpretation; and drafted the manuscript.

Ayush Batra assisted in the study design, collected the patient data, assisted in the study interpretation, revised the manuscript for critical intellectual content, and assisted in the study supervision.

Nathan A. Shlobin and Steven C. Hoffman collected the patient data, assisted in the study interpretation, and revised the manuscript for critical intellectual content.

Zachary S. Orban collected the patient data, assisted in the data management and data analysis, and revised the manuscript for critical intellectual content.

Igor J. Koralnik assisted in the study design, collected the patient data, assisted in the study interpretation, revised the manuscript for critical intellectual content, and assisted in the study supervision.

Eric M. Liotta conceived the study, led the study design, collected the patient data, performed the primary data analysis and interpretation, drafted the manuscript, supervised the study conduct, and took responsibility for the manuscript as a whole.

Funding Dr. Liotta is supported by National Institutes of Health grant L30 NS098427.

Data availability De-identified data and statistical code can be made available to appropriately qualified individuals for purposes of replication and at reasonable request.

\section{Declarations}

Ethics approval The study was approved by our institutional review board (STU00212627) with waiver of consent for retrospective analysis.
Consent for publication All authors have been included, and all authors have provided their approval for submission of the manuscript for consideration.

Competing interests The authors declare no competing interests.

\section{References}

1. WHO Coronavirus Disease (COVID-19) Dashboard. 2020. Accessed 2/24/2021, 2020, at https://covid19.who. int/

2. COVIDView: a weekly surveillance summary of U.S. COVID-19 activity. 2020. Accessed 11/23/2020, at https://www.cdc.gov/coronavirus/2019-ncov/covid-data/ pdf/covidview-11-20-2020.pdf

3. Emanuel EJ, Persad G, Upshur R, et al. Fair allocation of scarce medical resources in the time of Covid-19. N Engl J Med. 2020;382:2049-55.

4. Blumenthal D, Fowler EJ, Abrams M, Collins SR. Covid19 - implications for the health care system. N Engl J Med. 2020;383:1483-8.

5. Wadhera RK, Joynt Maddox KE, Kazi DS, Shen C, Yeh RW. Hospital revisits within 30 days after discharge for medical conditions targeted by the Hospital Readmissions Reduction Program in the United States: national retrospective analysis. BMJ. 2019;366:14563.

6. Arundel C, Lam PH, Khosla R, et al. Association of 30-day all-cause readmission with long-term outcomes in hospitalized older Medicare beneficiaries with heart failure. Am J Med. 2016;129:1178-84.

7. Lum HD, Studenski SA, Degenholtz HB, Hardy SE. Early hospital readmission is a predictor of one-year mortality in community-dwelling older Medicare beneficiaries. J Gen Intern Med. 2012;27:1467-74.

8. Courtney M, Edwards H, Chang A, Parker A, Finlayson $\mathrm{K}$, Hamilton K. Fewer emergency readmissions and better quality of life for older adults at risk of hospital readmission: a randomized controlled trial to determine the effectiveness of a 24-week exercise and telephone follow-up program. J Am Geriatr Soc. 2009;57:395-402.

9. Krumholz HM. Post-hospital syndrome-an acquired, transient condition of generalized risk. $\mathrm{N}$ Engl J Med. 2013;368:100-2.

10. Carfi A, Bernabei R, Landi F, Gemelli Against C-PACSG. Persistent symptoms in patients after acute COVID-19. JAMA 2020;324:603-5.

11. Goërtz YMJ, Van Herck M, Delbressine JM, Vaes AW, Meys R, Machado FVC, Houben-Wilke S, Burtin C, Posthuma R, Franssen FME, van Loon N, Hajian B, Spies Y, Vijlbrief H, van 't Hul AJ, Janssen DJA, Spruit MA. Persistent symptoms 3 months after a SARS-CoV-2 infection: the post-COVID-19 syndrome? ERJ Open Res. 2020 Oct 26;6(4):00542-2020. http://doi.org/10.1183/23120541. 00542-2020. PMID: 33257910; PMCID: PMC7491255. 
12. Rubin R. As Their Numbers Grow, COVID-19 "Long Haulers" Stump Experts. JAMA. 2020 Oct 13;324(14):1381-1383. http://doi.org/10.1001/jama.2020. 17709. PMID: 32965460.

13. Graham EL, Clark JR, Orban ZS, Lim PH, Szymanski AL, Taylor C, DiBiase RM, Jia DT, Balabanov R, Ho SU, Batra A, Liotta EM, Koralnik IJ. Persistent neurologic symptoms and cognitive dysfunction in non-hospitalized Covid-19 "long haulers". Ann Clin Transl Neurol. 2021 Mar 23. http://doi.org/10.1002/acn3.51350. Epub ahead of print. PMID: 33755344.

14. Liotta EM, Batra A, Clark JR, Shlobin NA, Hoffman SC, Orban ZS, Koralnik IJ. Frequent neurologic manifestations and encephalopathy-associated morbidity in Covid-19 patients. Ann Clin Transl Neurol. 2020 Nov;7(11):22212230. http://doi.org/10.1002/acn3.51210. Epub 2020 Oct 5. PMID: 33016619; PMCID: PMC7664279.

15. Robinson S, Howie-Esquivel J, Vlahov D. Readmission risk factors after hospital discharge among the elderly. Popul Health Manag. 2012;15:338-51.

16. Carey K, Lin MY. Hospital length of stay and readmission: an early investigation. Med Care Res Rev. 2014;71:99-111.

17. Felix HC, Seaberg B, Bursac Z, Thostenson J, Stewart MK. Why do patients keep coming back? Results of a readmitted patient survey. Soc Work Health Care. 2015;54:1-15.

18. Hernandez AF, Greiner MA, Fonarow GC, et al. Relationship between early physician follow-up and 30-day readmission among Medicare beneficiaries hospitalized for heart failure. JAMA. 2010;303:1716-22.

19. Somani SS, Richter F, Fuster V, et al. Characterization of patients who return to hospital following discharge from hospitalization for COVID-19. J Gen Intern Med. 2020;35:2838-44.

20. Atalla E, Kalligeros M, Giampaolo G, Mylona EK, Shehadeh F, Mylonakis E. Readmissions among patients with COVID-19. Int J Clin Pract. 2020 Sep 7:e13700. http:// doi.org/10.1111/ijcp.13700. Epub ahead of print. PMID: 32894801 .

21. Liotta EM, Batra A, Koralnik IJ. Reader Response: A Prospective Study of Neurologic Disorders in Hospitalized Patients With COVID-19 in New York City. Neurology. 2021 Mar 16;96(11):550. http://doi.org/10.1212/WNL.0000000000011613. PMID: 33723026.

22. Henricks K LA, Arenas I, Lewis DG. A tale of three cities: the state of racial justice in Chicago. Chicago IL: University of Illinois at Chicago, Institute for Research on Race and Public Policy; 2017.

23. Liotti FM, Menchinelli G, Marchetti S, Posteraro B, Landi F, Sanguinetti M, Cattani P. Assessment of SARS-CoV-2 RNA Test Results Among Patients Who Recovered From
COVID-19 With Prior Negative Results. JAMA Intern Med. 2021 May 1;181(5):702-704. http://doi.org/10.1001/ jamainternmed.2020.7570. PMID: 33180119; PMCID: PMC7662488.

24. Weitzer D and Richardson C. "Doctors examining possible link between chronic fatigue syndrome and COVID-19." ABC news; November 8, 2020. url: Doctors examining possible link between chronic fatigue syndrome and COVID-19 (msn.com). Accessed. January 10, 2021.

25. Mao L, Jin H, Wang M, et al. Neurologic manifestations of hospitalized patients with coronavirus disease 2019 in Wuhan. JAMA Neurol: China; 2020.

26. Romero-Sánchez CM, Díaz-Maroto I, Fernández-Díaz E, Sánchez-Larsen Á, Layos-Romero A, García-García J, González E, Redondo-Peñas I, Perona-Moratalla AB, Del Valle-Pérez JA, Gracia-Gil J, Rojas-Bartolomé L, Feria-Vilar I, Monteagudo M, Palao M, Palazón-García E, AlcahutRodríguez C, Sopelana-Garay D, Moreno Y, Ahmad J, Segura T. Neurologic manifestations in hospitalized patients with COVID-19: The ALBACOVID registry. Neurology. 2020 Aug 25;95(8):e1060-e1070. https://doi.org/10. 1212/WNL.0000000000009937. Epub 2020 Jun 1. PMID: 32482845; PMCID: PMC7668545.

27. Ellul MA, Benjamin L, Singh B, Lant S, Michael BD, Easton A, Kneen R, Defres S, Sejvar J, Solomon T. Neurological associations of COVID-19. Lancet Neurol. 2020 Sep;19(9):767-783. https://doi.org/10.1016/S14744422(20)30221-0. Epub 2020 Jul 2. PMID: 32622375; PMCID: PMC7332267.

28. Varatharaj A, Thomas N, Ellul MA, Davies NWS, Pollak TA, Tenorio EL, Sultan M, Easton A, Breen G, Zandi M, Coles JP, Manji H, Al-Shahi Salman R, Menon DK, Nicholson TR, Benjamin LA, Carson A, Smith C, Turner MR, Solomon T, Kneen R, Pett SL, Galea I, Thomas RH, Michael BD; CoroNerve Study Group. Neurological and neuropsychiatric complications of COVID-19 in 153 patients: a UK-wide surveillance study. Lancet Psychiatry. 2020 Oct;7(10):875-882. https://doi.org/10.1016/S22150366(20)30287-X. Epub 2020 Jun 25. Erratum in: Lancet Psychiatry. 2020 Jul 14;: PMID: 32593341; PMCID: PMC7316461.

29. Koralnik IJ, Tyler KL. COVID-19: a global threat to the nervous system. Ann Neurol. 2020 Jul;88(1):1-11. https:// doi.org/10.1002/ana.25807. PMID: 32506549; PMCID: PMC7300753.

Publisher's Note Springer Nature remains neutral with regard to jurisdictional claims in published maps and institutional affiliations. 